\title{
Melanocytic lesions associated with dermatofibromas: a spectrum of lesions ranging from junctional nevus to malignant melanoma in situ
}

\author{
Roy King ${ }^{1,2}$, Paul B Googe ${ }^{1,2,3}$, Robert N Page ${ }^{1,2}$ and Martin C Mihm Jr${ }^{4}$ \\ ${ }^{1}$ Knoxville Dermatopathology Laboratory, Knoxville, TN, USA; ${ }^{2}$ Department of Pathology, University of \\ Tennessee, Knoxville, TN, USA; ${ }^{3}$ Department of Pathology, Vanderbilt University, Nashville, TN, USA \\ and ${ }^{4}$ Department of Dermatopathology, Harvard Medical School, Massachusetts General Hospital, \\ Boston, MA, USA
}

\begin{abstract}
Dermatofibromas are common lesions that are often associated with epidermal hyperplasia and basal layer hyperpigmentation. A single case of lentiginous melanocytic hyperplasia overlying a dermatofibroma has been reported, however, nevi and melanoma have to the best of our knowledge, not been previously reported. We present 14 cases of melanocytic lesions associated with dermatofibromas. The clinical data and hematoxylinand eosin- stained sections were obtained and formalin-fixed, paraffin-embedded tissue was immunostained with antibodies against S-100, Mart-1, Factor XIIla, and CD117. There were nine females and five males ranging in age from 30 to 64 years and anatomic sites included back (five), arm (six), flank (two), and leg (one). The clinical diagnosis ranged from dermatofibroma to desmoplastic melanoma. Histologically, the melanocytic lesions included junctional, compound, and dermal nevi, and malignant melanoma in situ. In four cases the dermal component appeared to merge with the dermatofibroma. In the case of the melanoma in situ, the dermatofibroma abutted the epidermis. Immunohistochemically, the melanocytic lesions were S-100/ Mart-1 +, FXIIla-, and the dermatofibromas were S-100/Mart-1-, FXIIla +. Melanocytic neoplasia may appear in association with dermatofibromas. The fibrohistiocytic proliferation may be misinterpreted as a spindle or pleomorphic melanocytic process. Awareness of this association will aid in the correct diagnosis, and immunohistochemical studies will help in the differentiation of these two cell populations.
\end{abstract}

Modern Pathology (2005) 18, 1043-1047. doi:10.1038/modpathol.3800384; published online 1 April 2005

Keywords: dermatofibromas; melanoma; nevus

Dermatofibromas are common lesions that are often associated with a variety of epithelial changes ranging from pseudoepitheliomatous hyperplasia, to follicular basal cell hyperplasia, to squamous and basal cell carcinoma, among others. Pigmentary changes are often present clinically and histologically are usually represented by basal layer hyperpigmentation. A single case of lentiginous melanocytic hyperplasia overlying a dermatofibroma has been reported; however, melanocytic neoplasms occurring with dermatofibromas, have to the best of our knowledge, not been previously reported. We present the first series of cases in which both a

Correspondence: Dr R King, MD, Knoxville Dermatopathology Laboratory, 315 Erin Drive, Knoxville, TN 37919, USA.

E-mail: rking@labpath.com

Received 11 November 2004; revised and accepted 23 December 2004; published online 1 April 2005 melanocytic lesion and a dermatofibroma were present in the same biopsy. This report characterizes the clinically, histologically, and immunohistochemical findings in 14 such cases and addresses the potential pitfalls in the diagnosis of these lesions.

\section{Materials and methods}

In all, 14 cases of melanocytic lesions arising in association with dermatofibromas occurring over a period of 5 years were retrieved from the files of Knoxville Dermatopathology Laboratory. The clinical data were obtained and the hematoxylin- and eosin-stained sections were reviewed. In all cases, formalin-fixed, paraffin- embedded tissue was available for immunohistochemical staining. Antibodies to S-100 protein (polyclonal, 1:600, Dako Cytomation, Carpinteria, CA, USA), MART-1 (monoclonal, 
1:150, Dako Cytomation), factor XIIIa (polyclonal, 1:400, Dako Cytomation), and CD117 (polyclonal, 1:200, Biocare Medical, Walnut Creek, CA, USA). In all, 10 cases of dermatofibromas without melanocytic lesions were stained with CD117 (control group).

\section{Results}

\section{Clinical: (Table 1)}

There were nine females and five male patients ranging in age from 50 to 64 years (mean=to 50 years). Anatomical sites included arm (six), back (five), flank (two), and leg (one). The clinical diagnosis was variable and included dermatofibroma, nevus with cyst, basal cell carcinoma, dysplastic nevus, malignant melanoma, and desmoplastic melanoma.

\section{Histologic Findings}

Histologically, the melanocytic lesions were located directly over fibrohistiocytic proliferations. The dermatofibromas were centered on the dermis and were composed of a mix of fibroblast-like cells and histiocytes (some of which were multinucleate) in a storiform arrangement. The peripheries of the lesions were poorly demarcated, and there was wrapping of individual collagen fibers by fibroblastic cells. In all cases, there was accompanying epidermal hyperplasia with variable basal layer hyperpigmentation. The melanocytic lesions included a lentiginous junctional nevus (two), dermal nevus (three), compound nevus (eight) (Figure 1), and malignant melanoma in situ, superficial spreading type (one). In the cases where a junctional component was present, the melanocytic proliferation was associated with the epidermal hyperplasia.
In four cases, the dermal melanocytic component appeared to merge with the dermatofibroma (Figure 2). In the case of the malignant melanoma in situ, the dermatofibroma abutted the epidermis (Figure 3).

Immunohistochemically, the melanocytic lesions demonstrated positive staining for S-100 protein and MART-1, and negative staining for factor XIIIa. The dermatofibromas demonstrated a reverse staining pattern with factor XIIIa-positive staining and S100 protein and MART-1-negative staining (Figures 2 and 3). Staining for CD117 demonstrated diffuse strong staining within the dermatofibromas, within the overlying epidermis, and the melanocytic proliferations. In the control group of dermatofibromas, similar intense CD117 staining of the fibrohistiocytic proliferation and the overlying epidermis was noted, with no appreciable quantitative or qualitative staining difference compared with our dermatofibromas with melanocytic lesions.

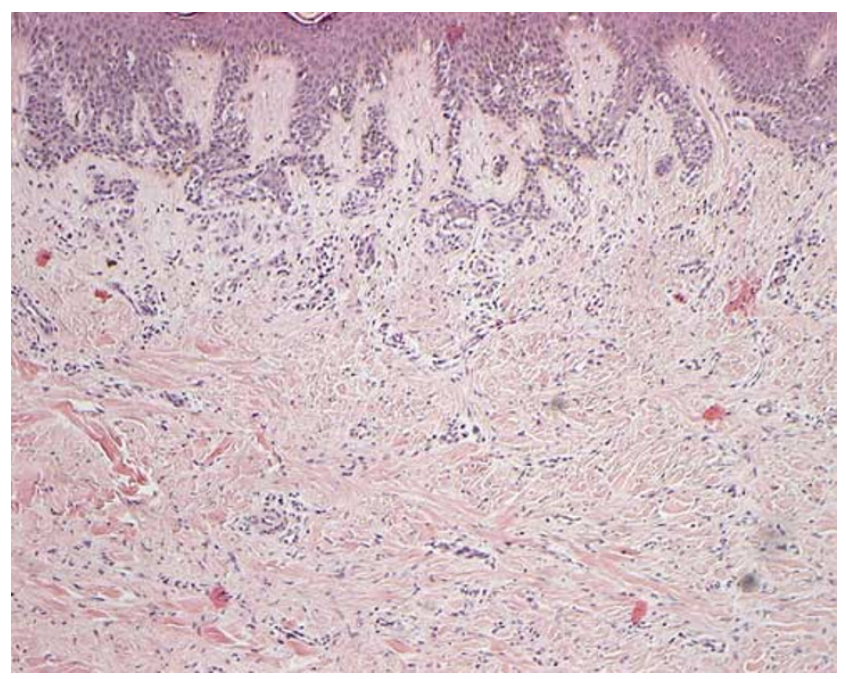

Figure 1 Compound nevus with underlying dermatofibroma.

Table 1 Clinical data

\begin{tabular}{llllll}
\hline Patient & Sex & Age & Site & Melanocytic lesion & Clinical \\
\hline 1 & F & 51 & Left lower leg & Junctional nevus & DF \\
2 & M & 39 & Upper mid-back & Junctional nevus & DF \\
3 & $\mathrm{~F}$ & 30 & Right flank & Compound nevus & Nevus with cyst \\
4 & $\mathrm{~F}$ & 47 & Right upper back & Dermal nevus & Nevus, rule out atypia \\
5 & $\mathrm{M}$ & 51 & Right upper back & Dermal nevus & Nevus, rule out atypia \\
6 & $\mathrm{~F}$ & 59 & Right arm & Dermal nevus & Nevus \\
7 & $\mathrm{~F}$ & 42 & Right upper posterior arm & Compound nevus & DF, rule out nevus \\
8 & $\mathrm{~F}$ & 50 & Right lateral torso & Compound nevus & Pigmented lesion, rule out desmoplastic MM \\
9 & $\mathrm{~F}$ & 59 & Left upper arm & Compound nevus & None given \\
10 & $\mathrm{~F}$ & 49 & Left upper forearm & Compound nevus & DF, rule out MM \\
11 & $\mathrm{M}$ & 52 & Left lower medial arm & Compound nevus & DF, rule out MM \\
12 & $\mathrm{M}$ & 64 & Right upper back & Compound nevus & SK, rule out BCC \\
13 & $\mathrm{~F}$ & 56 & Mid-upper back & Compound & Rule out dysplastic nevus \\
& & & & dysplastic nevus & \\
14 & $\mathrm{M}$ & 54 & Left upper arm & MMIS, SST & Rule out MM vs SK
\end{tabular}

$\mathrm{DF}=$ dermatofibroma; $\mathrm{BCC}=$ basal cell carcinoma; $\mathrm{MM}=$ malignant melanoma; MMIS, $\mathrm{SST}=$ malignant melanoma in situ, superficial spreading type; SK = seborrheic keratosis. 

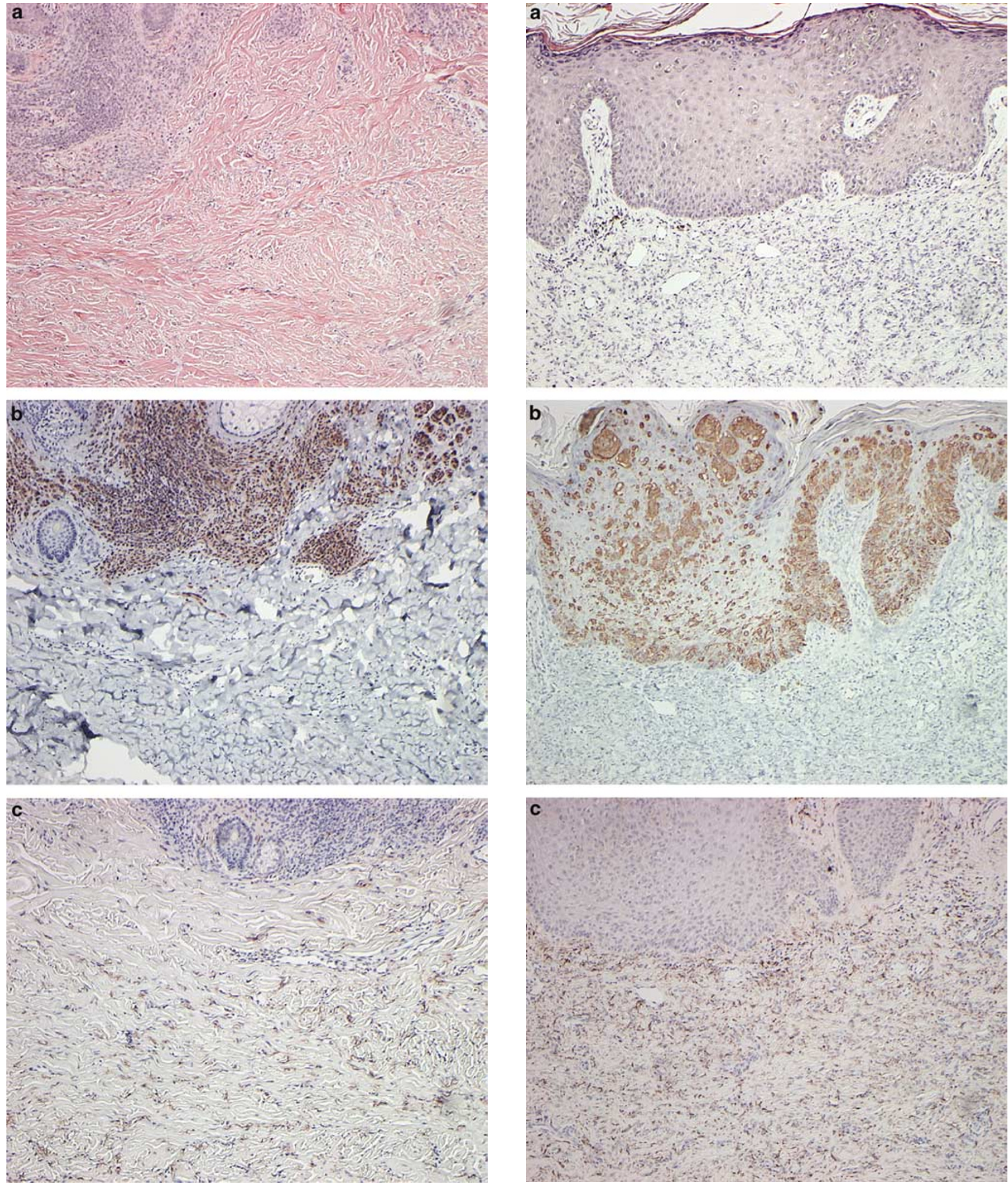

Figure 2 Dermal nevus with adjacent fibrohistiocytic proliferation (a). Mart-1 highlights the melanocytic proliferation (b), whereas Factor XIIIa highlights the fibrohistiocytic proliferation $(\mathbf{c})$.

Figure 3 Malignant melanoma in situ with the fibrohistiocytic proliferation extending up to the epidermis (a). Mart-1 stains melanoma cells in the epidermis but not the dermal proliferation (b), whereas Factor XIIIa has a reverse staining pattern highlighting the fibrohistiocytic proliferation, but not staining the melanocytic proliferation (c). 


\section{Discussion}

Dermatofibromas are common mesenchymal neoplasms, which have attracted a variety of names, including fibrous histiocytoma, sclerosing hemangioma, nodular subepidermal fibrosis, and histiocytoma. This variable nomenclature reflects the wide range of histologic appearances that these lesions may demonstrate. Epidermal changes overlying a dermatofibroma have been well documented, with acanthosis with basal layer hyperpigmentation occurring most frequently. ${ }^{1,2}$ Less commonly, a seborrheic keratosis-like pattern, and epidermal atrophy may also be seen overlying a dermatofibroma. ${ }^{2}$ Rarely, squamous cell carcinoma in situ ${ }^{3}$ and plate-like sebaceous hyperplasia ${ }^{4}$ may also occur. Focal acantholytic dyskeratosis, ${ }^{5}$ and epidermolytic hyperkeratosis $^{6}$ have also been described as coincidental findings. Basaloid proliferations overlying dermatofibromas may occur and these range from basal cell hyperplasia with follicular differentiation to well-developed basal cell carcinomas. ${ }^{7-11}$

Melanocytic proliferations occurring in association with dermatofibromas have been less well documented in the literature. In a report of three cases, Toda et $a l^{12}$ described three cases of lentiginous melanocytic hyperplasia overlying dermatofibromas and there was an increased frequency of S-100 protein and Mel-5 melanocytes along the basal layer of the epidermis overlying dermatofibromas. Along similar lines, it has been demonstrated that the number of tyrosinase immunopositive melanocytes in the pigmented dermatofibroma epidermis is increased up to two-fold when compared with the adjacent, nonlesional normal epidermis, and this may account for the hyperpigmentation seen clinically. ${ }^{13}$ To the best of our knowledge, there has been no report of melanocytic neoplasms arising in association with a dermatofibroma.

In our series, a variety of melanocytic neoplasms were present ranging from junctional nevus to melanoma in situ. In four cases of nevi with a dermal component, the melanocytes merged with the underlying fibrohistiocytic lesion, and in the case of melanoma in situ, the dermatofibroma abutted the epidermis, raising the possibility that the fibrohistiocytic portion of the lesion represented an unusual or atypical melanocytic proliferation. In particular, two benign melanocytic lesions have histologic features that resemble dermatofibroma, namely desmoplastic (sclerotic) nevus and amelanotic blue nevus. Harris et al, ${ }^{14}$ point out that in their experience, the most common pitfall was to mistake a desmoplastic nevus for a dermatofibroma, with both entities demonstrating epidermal hyperplasia and hyperpigmentation, hypercellular desmoplastic stroma, keloidal collagen, and multinucleated cells. However, in contradistinction to dermatofibromas, desmoplastic nevi, did not demonstrate adnexal induction and some cases demonstrated junctional and dermal nests of melanocytes, and all were S-100 immunopositive. In our series, all our cases had junctional and/ or dermal melanocytic nests and many did not demonstrate adnexal induction, rendering immunohistochemical studies the most reliable tool to separate these two entities. Similarly, amelanotic/ hypopigmented blue nevi may histologically be indistinguishable from dermatofibromas and Bhawan and Shuang-Lin, ${ }^{15}$ in their series of amelanotic blue nevi, indicate the most common pattern was the dermatofibroma-like pattern and only positive immunostaining with melanosome-associated antibody, MEL-5, confirmed the melanocytic nature of their lesions. In their series of hypopigmented blue nevi, Carr et al, ${ }^{16}$ also demonstrate the histologic similarities between their lesions and dermatofibromas and their lesions were all positive for S-100 and HMB-45, confirming their melanocytic lineage. Our cases raised the possibility of the dermatofibroma component representing part of combined common acquired nevus/blue nevus, however, the F XIIIa + / S-100, MART-1-immunophenotype confirmed the fibrohistiocytic nature of the DF component.

Of greater clinical importance, is the distinction of the fibrohistiocytic component from desmoplastic melanoma. Desmoplastic melanomas constitute a heterogeneous spectrum of lesions that may mimic a wide variety of lesions including dermatofibromas. ${ }^{17-19}$ Both these lesions may demonstrate a haphazard architecture containing hyperchromatic and sometimes bizarre nuclei; however, neurotropism and small foci of lymphoid aggregates are typically associated with desmoplastic melanomas. An atypical junctional melanocytic proliferation often accompanies desmoplastic melanomas, and is a useful feature indicating the melanocytic nature of these lesions, however, in our cases this was not helpful and in fact raised the possibility of an atypical dermal melanocytic proliferation. This was especially true in our case of melanoma in situ where the fibrohistiocytic proliferation raised the possibility of an associated desmoplastic melanoma. The absence of lymphoid follicles and lack of neurotropism were helpful negative histologic features, however, the immunohistochemical studies were most useful in separating out the lesions with the dermatofibromas being FXIIIa + /S-100-.

Nevi and dermatofibromas are both very commonly encountered lesions in dermatopathology and the presence of both lesions in one site may well be coincidental, and represent a 'collision type' lesion. However of interest, is the relationship of stem cell factor/c-kit to melanocytes, and has been implicated in the proliferation, survival, and migration of embryonic melanoblasts. ${ }^{20-21}$ Furthermore, fibroblastic tumor cell-derived stem cell factor may play an important role in melanocyte hyperplasia and hyperpigmentation in dermatofibromas. ${ }^{13}$ Utilizing c-kit (CD 117) immunostaining we did note significant staining of dermatofibromas $v s$ the adjacent nonlesional dermis, similar to a previous 
study. However, in our control group, similar findings were noted, and there were no quantitative differences in immunostaining in the dermatofibromas with and without melanocytic lesions. Our findings suggest that if these two lesions are causally related, other factors may be necessary to stimulate melanocyte proliferation.

In conclusion, we present a series of 14 cases of dermatofibromas associated with melanocytic lesions ranging from junctional nevus to melanoma in situ. Awareness of this phenomenon, together with appropriate immunohistochemical studies, will aid in the correct diagnosis of these cases and avoid the misinterpretation of the fibrohistiocytic lesions as unusual or atypical melanocytic proliferations.

\section{References}

1 Schoenfeld RJ. Epidermal proliferations overlying histiocytomas. Arch Dermatol 1964;90:266-270.

2 Halpryn HJ, Allen AC. Epidermal changes associated with sclerosing hemangiomas. Arch Dermatol 1959;80: 160-166.

3 Herman K, Kantor GR. Squamous cell carcinoma in situ overlying dermatofibroma. J Cutan Pathol 1990; 17:385-387.

4 Requena L, Roo E, Sanchez YE. Plate-like sebaceous hyperplasia overlying dermatofibromas. J Cutan Pathol 1992;19:253.

5 Ackerman AB, Hyman AB. Focal keratosis follicularis overlying dermatofibroma. Arch Dermatol 1971;104:219.

6 Ackerman AB. Dermatofibroma. In: Ackerman AB (ed). Histologic Diagnosis of Inflammatory Skin Diseases. Lea \& Febiger: Philadelphia, 1978, p 733.

7 Cheng L, Amini SB, Zaim MT. Follicular basal cell hyperplasia overlying dermatofibroma. Am J Surg Pathol 1997;21:711-718.

8 Bryant J. Basal cell carcinoma overlying long-standing dermatofibromas. Arch Dermatol 1977;113:1445-1446.
9 Goette DK, Helwig EB. Basal cell carcinomas and basal cell carcinoma-like changes overlying dermatofibromas. Arch Dermatol 1975;111:589-592.

10 Buselmeier TJ, Becker JH. Invasive basal cell carcinoma with metaplastic bone formation associated with a long-standing dermatofibroma. J Cutan Pathol 1979; 6:496.

11 McKenna KE, Somerville JE, Walsh MY, et al. Basal cell carcinoma occurring in association with dermatofibroma. Dermatology 1993;187:54-57.

12 Toda S, Heasley DD, Carlson JA, et al. Lentiginous melanocytic hyperplasia overlying dermatofibroma. J Dermatol 1996;23:840-844.

13 Shishido E, Kadono S, Manaka I, et al. The mechanism of epidermal hyperpigmentation in dermatofibroma is associated with stem cell factor and hepatocyte growth factor expression. J Invest Dermatol 2001;117:627-633.

14 Harris GR, Shea CR, Horenstein MG, et al. Desmoplastic (Sclerotic) nevus: an underrecognized entity that resembles dermatofibroma and desmoplastic melanoma. Am J Surg Pathol 1999;23:786-794.

15 Bhawan J, Shuang-Lin C. Amelanotic blue nevus: a variant of blue nevus. Am J Dermatopathol 1999;21: 225-228.

16 Carr S, Wilkinson B, Kossard S. Hypopigmented common blue nevus. J Cutan Pathol 1997;24:494-498.

17 Barnhill RL, Mihm Jr MC. The histopathology of cutaneous malignant melanoma. Semin Diagn Pathol 1993;10:47-75.

18 Bruijn JA, Mihm Jr MC, Barnhill RL. Desmoplastic melanoma. Histopathology 1992;20:197-205.

19 Carlson JA, Dickersin GR, Sober AJ, et al. Desmoplastic neurotropic melanoma: a clinicopathologic analysis of 28 cases. Cancer 1995;75:478-494.

20 Wehrle-Haller B. The role of Kit-ligand in melanocyte development and epidermal homeostasis. Pigment Cell Res 2003;16:287-296.

21 Hachiya A, Kobayashi A, Ohunchi A, et al. The paracrine role of stem cell factor/c-kit signaling in the activation of human melanocytes in ultravioletB-induced pigmentation. J Invest Dermatol 2001;116: 578-586. 